\title{
Diagnóstico del desarrollo de la destreza productiva del habla del idioma inglés de estudiantes de educación superior
}

Diagnosis of the development of productive English language skills of higher education students

\author{
Sonia Marcela Suarez Cabrera. ${ }^{1}$, Sandra Liliana Abarca Garcia. ${ }^{2}$, Ruth Patricia Molina
} Mazón. ${ }^{3}$ \& Maritza Lucía Larrea Vejar. ${ }^{4}$

\begin{abstract}
.
The objective of the present investigation was to diagnose the development of the productive English speaking skill of the students of the second semester of the Physical Culture Career of the National University of Chimborazo. For this purpose, a descriptive, qualitative-quantitative field research was applied through a sample represented by a control group of 50 students among men and women with heterogeneous knowledge of the English language. A peer-reviewed academic survey was applied to them, validated according to Cronbach's alpha. The survey contains questions with a Likert scale, which allowed the collection of data about criteria such as detecting student behavior, use of specific activities, use of strategies, new

\footnotetext{
${ }^{1}$ Universidad Nacional de Chimborazo, Riobamba, Ecuador, marcesu_23@ hotmail.com

${ }^{2}$ Escuela Superior Politécnica de Chimborazo, Facultad de Salud Pública, Riobamba, Ecuador, sandra.abarca@espoch.edu.ec

${ }^{3}$ Escuela Superior Politécnica de Chimborazo, Facultad de Ciencias, Riobamba, Ecuador, ruth.molina@espoch.edu.ec

${ }^{4}$ Escuela Superior Politécnica de Chimborazo, Facultad de Mecánica, Riobamba, Ecuador, maritza.larrea@espoch.edu.ec
} 
vocabulary, time spent, use of assistive technology instruments and assessment rubric. The information obtained was selected, tabulated, analyzed and interpreted through tables frequency graphs that allowed to identify that an intervention plan is required containing a strategy that enable us to improve the development of the productive skill of the English language speech, since problems of students ' behavior, incorrect application of class planning, complimentary use of technological tools and evaluation rubrics were detected.

Keywords: diagnosis, development, productive speech skills, english.

\section{Resumen.}

El objetivo de la presente investigación, fue diagnosticar el desarrollo de la destreza productiva del habla de inglés de los estudiantes del segundo semestre de la Carrera de Cultura Física de la Universidad Nacional de Chimborazo. Para ello, se aplicó una investigación de campo, descriptiva, cuali-cuantitativa a través de una muestra representada por un grupo de control de 50 estudiantes entre hombres y mujeres con conocimientos heterogéneos del idioma inglés. A quienes se les aplicó una encuesta revisada por pares académico expertos en el tema y validada según el alpha de Cronbach. La encuesta contiene preguntas con escala de Likert, que permitió recoger datos acerca de criterios como: detectar comportamiento del estudiante, uso de actividades específicas, uso de estrategias, vocabularios nuevos, tiempo de dedicación, uso de instrumentos tecnológicos de apoyo y rúbrica de evaluación. La información obtenida fue seleccionada, tabulada, analizada e interpretada a través de tablas y gráficos de frecuencias que permitieron identificar que se requiere de un plan de intervención que contenga una estrategia que permita mejorar el desarrollo de la destreza productiva del habla del idioma inglés, pues se detectaron problemas de comportamientos de los estudiantes, incorrecta aplicación de planificación de clases, uso complementario de herramientas tecnológicas y rubricas de evaluación.

Palabras claves: diagnóstico, desarrollo, destreza productiva del habla, inglés.

\section{Introducción.}

Para los estudiantes, hablar es lo que hacen cuando empiezan sus años de vida; pero la producción oral va más allá, porque desarrollan dos condiciones hablar y escuchar, es decir son complementarias. Pero cuando el estudiante las diferencia, puede practicar un discurso y también interactuar con otras personas. Con esta perspectiva, se orienta una clase de inglés enmarcando al proceso de enseñanza de una lengua extranjera al desarrollo, integración e interacción de factores culturales y significativos. 
Según Bygates (1991) la producción oral es la habilidad de articular oraciones en lo abstracto, que se crean y se acoplan a las circunstancias del momento, mediante la tomar decisiones rápidas y que se integran adecuadamente a contexto de problemas inesperados que se pueden presentar en diferentes tipos de conversación.

Por otra parte, Brown y Yule (1983), sostiene que la producción oral es un proceso interactivo que está compuesto de un significado con un canal donde se produce y recibe información por parte de participantes mediante sus experiencias, medio ambiente y propósito de la comunicación.

Los análisis de problemas de investigaciones, han servido como base sobre el uso de actividades, métodos participativos, estrategias didácticas comunicativas para el proceso de enseñanza aprendizaje durante la clase de inglés.

Maldonado y Romero (2011) a través de un estudio con enfoque cuali - cuantitativo, concluyeron que los docentes en el desarrollo de sus clases de inglés, al no usar un lenguaje claro con sus estudiantes en sus explicaciones, tuvieron el efecto de pérdida de interés para aprender el idioma Inglés, así mismo, se evidenció que el docente no aplicaba actividades interactivas en el momento de desarrollar la destreza de habla con sus estudiantes; es decir la carencia de enseñarles a establecer y mantener una conversación en Inglés, debido a la falta material didáctico y el uso único de una sola actividad para promover la expresión oral.

Para los docentes es un desafío motivar y enseñarles a hablar a los estudiantes, ya que involucra factores como el uso correcto del idioma y espontaneidad al expresarse, El docente debe evitar que el estudiante al momento de hablar en inglés, transmita mensajes erróneos y descontextualizados hacia el receptor u oyente. Es decir, debe guiar al estudiante en el uso apropiado de la oralidad para una efectiva comprensión del mensaje que este compuesto de pensamientos, sentimientos y un acervo cultural de acorde y de importancia entre los interlocutores (Grau-Tarruell, et al., 2005). Es decir, objetivo principal, es guiar a los estudiantes a comunicarse en tiempo real en situaciones específicas.

Según Roach (2010) el desarrollo de la destreza speaking se desarrolla en cinco etapas: respiración, fonación, resonancia, articulación e integraciones neurológicas. Sostiene que la pronunciación se caracteriza por la forma con que se producen los sonidos de las frases, 
expresiones y palabras. En el contexto del idioma inglés, los sonidos se analizan por el acento, entonación y ritmo que determinan el éxito de la comunicación a través de las interacciones.

Según Cenoz (2010), sostiene que uno de los conceptos más relevantes en lingüística aplicada fueron las competencias comunicativas, debido a su importancia en el estudio de la adquisición de una segunda lengua, como a nivel práctico de la enseñanza del idioma; pues las competencias comunicativas fueron un concepto determinante para entender las respuestas al objetivo de la enseñanza de lenguas, al saber sobre los conocimientos, capacidades o destrezas que se necesitaban para hablar una lengua, o en qué consiste adquirir una lengua.

Fernández (2008) resalta en los estudiantes, su forma espontánea de usar las ideas para entablar sus propias conversaciones, desarrollando sus propias habilidades de producción oral, por lo que se pueden derivar en varias actividades; entre ellas: una conversación con un guión previamente escrito, ideas antes de una participación en una conversación, dramatizaciones, trabajos individuales o en grupos que permiten a interactuar y ayudar a los participantes a un trabajo colaborativo.

En el contexto del aprendizaje y uso de la lengua del idioma inglés, los estudiantes de forma individual o grupal, han desarrollado competencias generales y comunicativas, que han sido utilizándolas de acuerdo a sus restricciones, contexto y condiciones para ejecutar tareas propias del idioma a través de la aplicación de estrategias apropiadas. En el marco de las competencias comunicativas, el concepto de destreza o habilidad lingüística tomó gran relevancia, mismas que fueron inducidas y fueron puestas en práctica por los estudiantes en los procesos comunicativos. Pérez M. I., (2015) argumenta que el hablar y el entender fueron destrezas básicas de la comunicación oral; mientras que las destrezas básicas de la comunicación escrita fueron el escribir y el leer.

Alfaro (2012) indica que la habilidad de escuchar en un estudiante, es una acción de poner atención percibida mediante el oído, ya que es importante que el mensaje transmitido debe ser comprendió a fin de no tergiversarlo y otorgarle su adecuada valoración. Por otra parte, se considera la destreza de hablar, a la intención que tiene un estudiante para comunicarse y 
manifestar sus opiniones sobre un tema determinado, diciendo palabras fáciles de entender a fin de dar un mensaje de forma natural.

Batista \& Romero (2007) indica que hablar con claridad es una destreza que consiste en saber decir algo, involucrando la forma de cómo se habla, cómo se utiliza la expresión oral, ya que cuando se manifiesta se pone en evidencia la personalidad, que identifica el patrón por cual se juzgó, se aceptó o rechazó. En síntesis, es importante que al hablar se debe demostrar ser convincente, ordenado, claro, transmitir entusiasmo y ser capaz de persuadir para ser escuchado.

Según Kremers (2000) las competencias comunicativas estaban compuestas por componentes que consideran las destrezas parciales; tales como: competencia gramatical (conocimientos de formas y contenidos), habilidades verbales y no verbales (recurren el interlocutor a la hora de hacer uso de su creatividad para solucionar posibles problemas de discurso). Se consideró relevante su integración, ya que partieron de la hipótesis, de que las estrategias se aprendieron por la práctica y auto experimentación en situaciones reales o comunicativas.

Se considera un diálogo, a la conversación que pueden entablar dos o más individuos, quienes exponen sus ideas de modo alternativo con el fin de exponer sus posturas. Es decir, un diálogo también es una discusión cuyo desenlace tiene el propósito de llegar a un acuerdo

Vidoza, (2012) considera que la entrevista como un diálogo, donde el entrevistador, plantea preguntas a el "entrevistado", con el objetivo de de obtener información. Es decir son dos personas intercambiando mensajes, donde se generan una diversidad de preguntas y con sus respectivas respuestas dentro de un contexto, tratando de lograr como propósito final la obtención de la información.

Por otra parte, el debate se deriva de un acto propio de la comunicación humana, ya que consiste en una discusión de un tema polémico denominado premisa o moción que se puede dar entre dos o más grupos de personas. Es de carácter argumentativo, y está guiado por un moderador. El objetivo del debate es expresar ideas para argumentarlas, y la forma de responderlas es mediante la contra argumentación. Sin duda, las redes sociales explotan esta técnica, (Gonzáles Paz, 2014). 


\section{Metodología.}

La investigación tiene carácter educativo, orientada a la producción del conocimiento a fin de establecer una línea base o estudio modelo para arrancar investigaciones experimentales, donde se aplique un método científico hipotético deductivo.

Al ser una investigación de tipo descriptiva, tuvo que adaptarse a un entorno educativo para contribuir y transformar el sistema social que desarrolla procesos que necesitan ser modificados, actualizados mediante alternativas o estrategias didácticas que permitan mejorar el proceso de enseñanza aprendizaje.

La investigación tuvo enfoque cuali-cuantitativo, a través de una investigación bibliográfica, que contiene su revisión de literatura de previas investigaciones con fuentes primarias y secundarias sostenidas experimentos, resultados, teorías, metodologías, estrategias, propuestas, hipótesis, técnicas e instrumentos.

Por otra parte, al ser una investigación descriptiva, se realizó el análisis interpretativo de los datos obtenidos, a través de la tabulación numérica y el análisis estadístico de las respuestas proporcionadas por los estudiantes que conforman la muestra de la investigación mediante un instrumento validado.

El diseño de la investigación fue encaminado al diagnóstico de como los estudiantes desarrollaron la destreza productiva del habla del idioma inglés que representan un grupo de control y a quienes se aplicó un instrumento de recolección de la información.

La investigación fue de campo, y los datos se obtuvieron con los actores del objeto de estudio de forma directa y confiable, es decir para la investigación se consideró una muestra intencional conformada por 50 estudiantes del segundo semestre de la Carrera de Cultura Física de la Universidad Nacional de Chimborazo, entre hombres y mujeres que tienen un nivel homogéneo de conocimiento que siguen un plan analítico del idioma inglés.

El investigador utilizó la técnica de la encuesta para la recolección de la información, mientras que el instrumento fue creado de forma estructurada por el autor de la investigación y al mismo tiempo revisada y aprobada por pares académicos expertos del área de inglés. El instrumento fue sometido al alpha de cronbach con el fin de determinar su confiabilidad, el 
mismo que obtuvo un valor de 0.82 pues estaba adaptado al objetivo del contexto de la investigación.

Las dimensiones consideradas para obtener la línea base de la investigación fueron determinadas por: comportamiento del estudiante, uso de actividades específicas, uso de estrategias, vocabularios nuevos, tiempo de dedicación, uso de instrumentos tecnológicos de apoyo y rúbrica de evaluación que deben ser considerados dentro del desarrollo de la destreza productiva del habla de inglés

La encuesta utilizada para la obtención de la información y que aplicada a la muestra esta estructura por ocho preguntas con una escala de Linkert con opciones cerradas de respuesta de selección: Siempre, A veces y Nunca. Los contextos de las preguntas abarcan las dimensiones del objeto de estudio que fueron validados por expertos y que dieron su certificación de que las preguntas son claras y coherentes. Por otra parte, las opciones de las preguntas son de tipo cualitativas pero una vez tabuladas fueron tabuladas y procesadas.

Con la aplicación de la encuesta, se obtuvo un diagnóstico detallado de la opinión de los encuestados en referencia al desarrollo de la destreza productiva del habla del idioma inglés. Permitiendo que los datos obtenidos puedan ser seleccionados, tabulados, organizados y procesados de forma descriptivas con distribución de frecuencias y porcentajes.

Para obtener el procesamiento de los datos se procedió de la siguiente manera:

- Las opciones de las preguntas fueron elaboradas con la escala de Likert (siempre, A veces, Nunca)

- Se aplicó la encuesta a la muestra de la investigación durante un periodo inicial

- La información obtenida de la encuesta, fue seleccionada, organizada e preparada para el ingreso mediante tablas de información.

- Los datos obtenidos fueron ingresados en una hoja de cálculo de Microsoft Excel a través de tablas para su cálculo de frecuencias y generación de gráficos.

- Los datos calculados fueron analizados de forma descriptiva, es decir se procedió al análisis e interpretación de los resultados obtenidos. 
- Finalmente, del análisis e interpretación de los resultados se plantearon las conclusiones del contexto de la investigación.

\section{Resultados.}

Pregunta 1. ¿En las clases, la docente aplicó actividades como diálogos, conversaciones para mejorar la producción oral?

Tabla 1. La docente aplicó actividades como diálogos, conversaciones

\begin{tabular}{lcc}
\hline Variable & $\mathbf{N}^{\mathbf{0}}$ Estudiantes & $\mathbf{\%}$ \\
\hline Siempre & 17 & 34 \\
A Veces & 30 & 60 \\
Nunca & 3 & 6 \\
Total & 50 & $\mathbf{1 0 0}$ \\
\hline
\end{tabular}

Fuente: Instrumento de obtención de datos.

Elaborado por: Sonia Suárez, 2016.

Análisis e interpretación: Del 100\% de los estudiantes encuestados sobre el contexto de la investigación, el 34\% respondieron que la docente Siempre aplicó actividades como diálogos, conversaciones para mejorar la producción oral en el aula, el 60\% respondió que A veces, mientras que el 6\% respondió que Nunca. Por lo que, se debe tomar atención en este criterio, pues la mayoría de los estudiantes sostienen que se debe hacer énfasis en la aplicación de diálogos y conversaciones a fin de mejorar la producción oral en el idioma inglés; es decir, se debe analizar el uso alternativo de métodos y técnicas en el proceso de aprendizaje del idioma Inglés identificando que: tan solo el 34\% indica que aplica actividades como diálogos, conversaciones para mejorar la producción oral, pues al existir deficiencias en el aprendizaje del idioma Inglés reflejando en el poco interés para desarrollar las habilidades de producción oral.

Pregunta 2. ¿Teme ser criticado por sus compañeros en el aula, si comete errores al hablar en el idioma inglés? 
Tabla 2. Fue criticado al cometer errores al hablar en el idioma inglés.

\begin{tabular}{ccc}
\hline Variable & $\mathbf{N}^{\mathbf{0}}$ Estudiantes & $\mathbf{\%}$ \\
\hline Siempre & 30 & 60 \\
A Veces & 20 & 40 \\
Nunca & 0 & 0 \\
Total & $\mathbf{5 0}$ & $\mathbf{1 0 0}$ \\
\hline
\end{tabular}

Fuente: Instrumento de obtención de datos.

Elaborado por: Sonia Suárez, 2016.

Análisis e Interpretación: Del 100\% de los estudiantes encuestados sobre el contexto de la investigación, el $60 \%$ respondieron que Siempre temen ser criticados por sus compañeros cuando cometen errores al momento de hablar en el idioma Ingles, el 40\% respondieron A veces; mientras que no se registraron frecuencias de la opción Nunca. Es decir, el 100\% de los estudiantes son afectados por diversos factores cuando desarrollan actividad de diálogos en el idioma inglés; por lo que es necesario buscar una estrategia con actividades que permitan fortalecer la autoestima e incentivarle a la participación activa en el aula.

Pregunta 3. ¿Si se equivocó al pronunciar frases con vocabulario nuevo en inglés, la docente lo corrigió?

Tabla 3. Le corrigió cuando usted se equivocó al pronunciar frases.

\begin{tabular}{lcc}
\hline Variable & $\mathbf{N}^{\mathbf{0}}$ Estudiantes & $\mathbf{\%}$ \\
\hline Siempre & 32 & 64 \\
A Veces & 17 & 34 \\
Nunca & 1 & 2 \\
Total & $\mathbf{5 0}$ & $\mathbf{1 0 0}$
\end{tabular}

Fuente: Instrumento de obtención de datos.

Elaborado por: Sonia Suárez, 2016.

Análisis e interpretación: Del 100\% de los estudiantes encuestados sobre el contexto de la investigación, el 64\% respondió que la docente Siempre corrigió al estudiante cuando se equivocaron al pronunciar frases con nuevo un vocabulario al hablar en el idioma inglés, el $34 \%$ respondió que A veces; mientras que el 2\% respondió que Nunca. Es decir, la mayoría 
de los estudiantes sostiene que fueron corregidos al pronunciar vocabulario nuevo, por lo que se requiere elaborar material activo y dinámico con el fin de que los estudiantes puedan interactuar y corrijan errores de pronunciación en las conversaciones o diálogos en inglés.

Pregunta 4. ¿Tuvo Usted la oportunidad de practicar el nuevo vocabulario que aprendió en el aula después de clases?

Tabla 4. Practicó el nuevo vocabulario.

\begin{tabular}{ccc}
\hline Variable & $\mathbf{N}^{\mathbf{0}}$ Estudiantes & $\mathbf{\%}$ \\
\hline Siempre & 11 & 22 \\
A Veces & 21 & 42 \\
Nunca & 18 & 36 \\
Total & $\mathbf{5 0}$ & $\mathbf{1 0 0}$ \\
\hline
\end{tabular}

Fuente: Instrumento de obtención de datos.

Elaborado por: Sonia Suárez, 2016.

Análisis e Interpretación: Del 100\% de los estudiantes encuestados sobre el contexto de la investigación, el $22 \%$ respondió que tuvieron la oportunidad de practicar el nuevo vocabulario aprendido después de la clase, el $42 \%$ respondieron que A veces; mientras que el 36\% respondieron que Nunca practicaron lo aprendido en el aula. Es decir que la mayoría de los estudiantes no practican el nuevo vocabulario fuera de la clase, pues solo la minoría son los que practicaban el vocabulario aprendido por diferentes razones. Por lo que se requiere elaborar material complementario para que practiquen lo aprendido desde casa.

Pregunta 5. ¿Considera que, al utilizar mayor cantidad de tiempo a la práctica de la habilidad oral en el aula, se mejora el manejo del idioma inglés?

Tabla 5. Práctica de la habilidad oral en el aula.

\begin{tabular}{lcl}
\hline Variable & $\mathbf{N}^{\mathbf{o}}$ Estudiantes & $\mathbf{\%}$ \\
\hline Siempre & 6 & 12 \\
A veces & 18 & 36 \\
Nunca & 26 & 52 \\
Total & $\mathbf{5 0}$ & $\mathbf{1 0 0}$ \\
\hline
\end{tabular}


Fuente: Instrumento de obtención de datos.

Elaborado por: Sonia Suárez, 2016.

Análisis e Interpretación: Del 100\% de los estudiantes encuestados sobre el contexto de la investigación, el 12\% respondió que la docente Siempre utilizó dramatizaciones para mejorar la producción oral, el 36\% de los estudiantes respondieron que A veces; mientras que el 52\% respondió que Nunca la docente aplicó dramatizaciones en el aula. Es decir que la mayoría de los estudiantes sostienen que la docente no utiliza la mayor cantidad de tiempo a la práctica de la habilidad oral, mientras que solo la minoría sostiene lo contrario. Por lo que se requiera la elaboración de material para que los estudiantes dramaticen temas determinados a fin de lograr un aprendizaje significativo.

\section{Pregunta 6. ¿Considera que al aplicar actividades tales como discusiones, dramatizaciones se mejora la habilidad productiva oral en el idioma inglés?}

Tabla 6. El aplicar actividades en el aula mejoró su habilidad productiva oral.

\begin{tabular}{ccc}
\hline Variable & $\mathbf{N}^{\mathbf{0}}$ Estudiantes & $\mathbf{\%}$ \\
\hline Siempre & 33 & 66 \\
A Veces & 17 & 34 \\
Nunca & 0 & 0 \\
Total & $\mathbf{5 0}$ & $\mathbf{1 0 0}$ \\
\hline
\end{tabular}

Fuente: Instrumento de obtención de datos.

Elaborado por: Sonia Suárez, 2016.

Análisis e Interpretación: Del 100\% de los estudiantes encuestados sobre el contexto de la investigación, el 66\% respondió Siempre al aplicar actividades tales como discusiones, dramatizaciones se mejora la habilidad productiva oral en el idioma inglés, el 34\% respondió que A veces; mientras que no se registraron frecuencias de la opción Nuca. Por lo que la mayoría de los estudiantes, consideran que para mejorar la habilidad productiva oral del idioma inglés se debe hacer énfasis en el uso de discusiones, dramatizaciones como estrategia en el aula. Por otro lado, ningún estudiante se opone al uso de este tipo de actividades para mejorar la habilidad de la productividad oral. 
Pregunta 7. ¿Cree Usted, que su conocimiento del idioma inglés le permitió construir conversaciones, diálogos en el aula sin requerir de apoyo tecnológico?

Tabla 7. El conocimiento del idioma inglés permitió construir conversaciones, diálogos sin apoyo tecnológico.

\begin{tabular}{ccc}
\hline Variable & $\mathbf{N}^{\mathbf{0}}$ Estudiantes & $\mathbf{\%}$ \\
\hline Siempre & 24 & 48 \\
A Veces & 25 & 50 \\
Nunca & 1 & 2 \\
Total & $\mathbf{5 0}$ & $\mathbf{1 0 0}$ \\
\hline
\end{tabular}

Fuente: Instrumento de obtención de datos.

Elaborado por: Sonia Suárez, 2016.

Análisis e interpretación: Del 100\% de los estudiantes encuestados sobre el contexto de la investigación, el $48 \%$ respondió que Siempre su conocimiento permitió construir conversaciones sin la necesidad de requerir apoyo tecnológico, el 50\% indico que A veces; mientras que el $2 \%$ respondió que nunca. Es decir, que el criterio de requerir apoyo tecnológico, para entablar diálogos o conversaciones se convierte en una necesidad para hablar en el idioma inglés. Por lo que se necesita adoptar una alternativa de estudio del aprendizaje del idioma inglés que incluyan herramientas de la web 2.0 como: traductor de texto para escuchar, reconocer y ambientarse con las palabras de inglés de una manera que el recurso está al alcance y disponibilidad del estudiante.

Pregunta 8. ¿La docente evaluó las actividades orales realizadas en clase?

Tabla 8. La docente evaluó las actividades orales

\begin{tabular}{lcc}
\hline Variable & $\mathbf{N}^{\mathbf{0}}$ Estudiantes & $\mathbf{\%}$ \\
\hline Siempre & 20 & 40 \\
A Veces & 30 & 60 \\
Nunca & 0 & 0 \\
Total & $\mathbf{5}$ & $\mathbf{1 0 0}$ \\
\hline
\end{tabular}

Fuente: Instrumento de obtención de datos.

Elaborado por: Sonia Suárez, 2016. 
Análisis e interpretación: Del 100\% de los estudiantes encuestados sobre el contexto de la investigación, el $40 \%$ respondió que Siempre la docente evaluó las actividades orales realizadas en clases, el $60 \%$ respondió que A veces; mientras que no se registran frecuencias con respecto a la opción Nunca. Es decir, la mayoría considera que la docente debe evaluar dichas actividades a fin de evidenciar, detectar y dar seguimiento de la destreza de cada estudiante con respecto a la habilidad productiva del habla del idioma inglés. Además, se indica que las clases son más teóricas que prácticas, por ende, los estudiantes son más propensos cometer errores y sentir temor en sus participaciones, más aún cuando se practicaba un nuevo vocabulario, dando como resultado un escaso desarrollo de la destreza productiva del habla del idioma inglés y falta de evidencias de evaluación y desarrollo de la destreza.

\section{Conclusiones:}

- A través del diagnóstico se detectó que se requiere del uso de métodos o estrategias innovadoras que motiven en los estudiantes de del segundo semestre de la Carrera de Cultura Física de la Universidad Nacional de Chimborazo el desarrollo de la destreza productiva del habla de inglés, pues no existen actualmente actividades dinámicas dentro de un plan de intervención que permita que los estudiantes no sientan temor por equivocarse al momento de mantener un dialogo en inglés, es decir se requiere realizar actividades motivadoras que pueden ser planteadas con el uso de la tecnología que brindan las herramientas web 2.0.

- Durante el desarrollo de las actividades aplicadas en la destreza productiva del habla de inglés, se requiere aplicar una rúbrica de evaluación que considere instrumentos de evaluación que califiquen la participación de los estudiantes durante actividades tales como diálogos, conversaciones, entrevistas; a fin de monitorear y registrar el progreso de los participantes.

- El diseño de una propuesta a través de un conjunto de actividades que cubra la problemática encontrada en el actual estudio, será un instrumento que se pueda integrar en una investigación con método científico, a fin de establecer incidencia o mejora en el desarrollo de la expresión oral. 


\section{Referencias bibliográficas.}

Alfaro, S. (9 de Abril de 2012). Habilidades Comunicativas. Obtenido de Concepto de las Habilidades Comunicativas: http://ejerciciohabilidadescomunicativas.blogspot.com/2012/04/concepto-de-lashabilidades.html

Brown, G. y Yule, G. (1983). Teaching the spoken language. New York: Cambridge

University Press

Batista, J., \& Romero, M. (2007). Habilidades comunicativas del líder en universidades privadas. Laurus : Revista de Educación, 36-64, ISBN 1315-883X.

Bygates, M. (1991), Speaking. Reino Unido: Oxford University Press

Cenoz, L. (2010). El concepto de competencia comunicativa. Revista de Enfoque comunicativo, 1-10.

Fernàndez, M. (2008). Mejorar laproducciòn oral de estudiantes de inglès como lengua extranjera en elcentro de idiomas de UABC- Tijuana. Ponencia presentada en VII encuentro nacional e Internacional de Centros de Autoacceso, 1-20.

Gonzáles Paz, J. (30 de Septiembre de 2014). Qué es un debate y cuáles son sus características. Obtenido de Educacion niños: http://www.guioteca.com/educacion-para-ninos/que-es-un-debate-y-cuales-son- suscaracteristicas/

Grau-Tarruell, M, Palou-Sangrà, J, Vilà-Santasusana, M, Ballesteros-Gómez, C, CastellàLidon, J, \& Cros Alavedra, A. (2005). El discurso oral formal: Contenidos

Kremers, M. (2000). EL USO DE LAS ESTRATEGIAS DE APRENDIZAJE EN LA EXPRESIÓN ORAL. Obtenido de Conceptializacion: http://cvc.cervantes.es/ensenanza/biblioteca_ele/asele/pdf/11/11_0461.pdf

Maldonado, M. D., \& Romero, M. J. (2011). Las actividades interactivas que utilizan los docentes de inglés para el desarrollo de la destreza de hablar en los décimos años de educación básica del instituto superior Daniel Reyes, Colegio Víctor Mideros, colegio nacional san pablo y colegio anexo al ISPED Instituto Alfredo Pérez Guerrero [Pregrado, UNIVERSIDAD TÉCNICA DEL NORTE]. http://repositorio.utn.edu.ec/bitstream/123456789/2042/1/05\%20FECYT\%201181\% 20T ESIS.pdf

Pérez, M. I. (2015). Procesos de aprendizaje: Desarrollo de habilidades comunicativas. Grado en magisterio de Educacion Primaria, 1-3. 
Roach, P. (2010). English Phonetics and Phonology Fourth Edition: A Practical Course. [Fonética inglesa y fonología Cuarta edición: un curso práctico]. Cambridge University Press

Vidoza, J. (2012). La entrevista. Obtenido de Características generales de la entrevista: http://tecnologiaedu.us.es/formate/curso/modulo9/412caracteristicas.htm

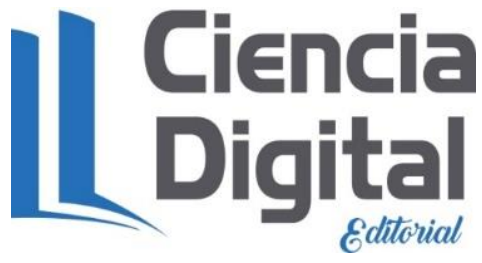




\section{PARA CITAR EL ARTÍCULO INDEXADO.}

Suarez Cabrera, S. M., Abarca Garcia, S. L., Molina Mazón, R. P., \& Larrea Vejar, M. L. (2020). Diagnóstico del desarrollo de la destreza productiva del habla del idioma inglés de estudiantes de $\begin{array}{lllll}\text { educación } & \text { superior. } & \text { Explorador } & \text { Digital, } & 4(3),\end{array}$ https://doi.org/10.33262/exploradordigital.v4i3.1316

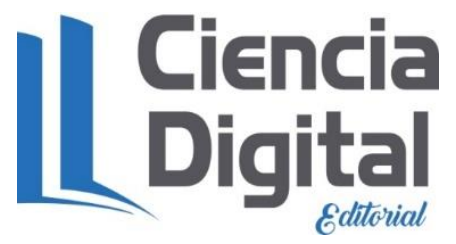

El artículo que se publica es de exclusiva responsabilidad de los autores y no necesariamente reflejan el pensamiento de la Revista Explorador Digital.

El artículo queda en propiedad de la revista y, por tanto, su publicación parcial y/o total en otro medio tiene que ser autorizado por el director de la Revista Explorador Digital.
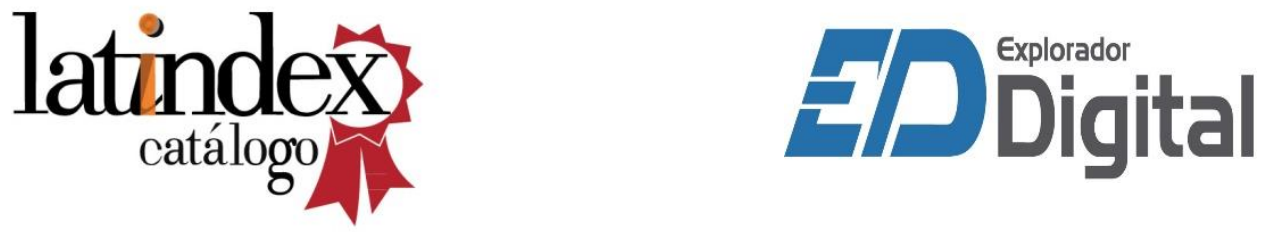